\title{
Stewart-Treves Syndrome in Obesity-Associated Chronic Lymphedema: A Case Report
}

\author{
Michael J. Chaney ${ }^{\mathrm{a}, \mathrm{d}}$, Xuanzhen Piao ${ }^{\mathrm{a}}$, Nayha Tahir ${ }^{\mathrm{b}}$, Grace W. Ying ${ }^{\mathrm{b}}$, \\ Yetunde Omotosho ${ }^{\mathrm{b}}$, Aneeba Farooqi ${ }^{\mathrm{b}}$, Farah Zahra ${ }^{\mathrm{c}}$
}

\begin{abstract}
Stewart-Treves syndrome (STS) is defined as the development of cutaneous angiosarcoma in the presence of long-standing lymphedema and is a rare disease with only about 400 cases reported in world literature. We report a case of a 63-year-old morbidly obese woman with a long-standing history of lymphedema who developed angiosarcoma of the right lower extremity with metastasis and presented with acute respiratory distress. The patient underwent a thorough laboratory workup with a chest X-ray showing bilateral effusions. The hematology-oncology service was consulted and found the patient to have significant progression of angiosarcoma causing respiratory failure and cardiac instability. A decision to transition to hospice care was made and the patient eventually passed away in the intensive care unit. We present this case to raise awareness of STS in medical literature to understand its clinical manifestations better. Early detection is imperative as angiosarcoma is commonly an aggressive disease.
\end{abstract}

Keywords: Stewart-Treves; Cancer; Obesity; Histology

\section{Introduction}

Clinical presentation can vary, but Stewart-Treves syndrome (STS) typically presents with a purple patch that develops into a nodule on the skin. In 1948, Stewart and Treves first described the development of angiosarcoma in women following radical mastectomy for breast cancer complicated by edema [1]. STS is typically found in an ipsilateral upper extremity post radical mastectomy complicated by lymphedema.

Manuscript submitted August 8, 2021, accepted August 28, 2021

Published online September 29, 2021

${ }^{a}$ Chicago Medical School, Rosalind Franklin University of Medicine and Science, North Chicago, IL, USA

${ }^{b}$ Chicago Medical School Internal Medicine Residency Program at Northwestern Mchenry Hospital, McHenry, IL, USA

'Department of Internal Medicine, Northwestern Medicine McHenry Hospital, McHenry, IL, USA

${ }^{\mathrm{d} C o r r e s p o n d i n g ~ A u t h o r: ~ M i c h a e l ~ C h a n e y, ~ C h i c a g o ~ M e d i c a l ~ S c h o o l, ~ R o s a l i n d ~}$ Franklin University of Medicine and Science, North Chicago, IL, USA.

Email: mchaney1124@gmail.com

doi: https://doi.org/10.14740/jmc3769
In this case report, our patient who had STS in the right lower extremity in the presence of obesity-associated lymphedema is atypical. The average life expectancy of cutaneous angiosarcoma is 2.5 years with most patients dying within the first 2 years from metastatic disease [2]. Untreated patients, on average, live 5 - 8 months [3]. These factors make early detection of STS paramount as the treatment before metastasis via wide excision tissue removal is the best option in improving outcomes.

\section{Case Report}

\section{Investigations}

The patient was a 63-year-old woman with a past medical history of lymphedema of the right lower extremity for the past 2 years and diagnosis of angiosarcoma of the right lower extremity status post right above knee amputation. The patient also had surgical re-vision of the right knee stump due to recurrent disease. Ultimately there was metastasis to lymph nodes, bone, lung, and adrenal glands status post radiation and chemotherapy. Other history included recent right lower lobe segmental pulmonary embolism and bilateral lower extremity deep vein thrombosis treated with rivaroxaban, recent resolution of a large right-sided pneumothorax, morbid obesity for her entire recorded adult life with body mass index (BMI) of 41.6, hypertension, type 2 diabetes mellitus, hyperlipidemia, and no other surgical history. The patient was admitted to the intensive care unit (ICU) from an inpatient rehabilitation facility for worsening shortness of breath. She was found to be in atrial fibrillation and respiratory distress. A review of systems was unable to be obtained due to the patient's mental status.

Of note, the patient had an extensive history of radiation and chemotherapy for metastatic angiosarcoma of the right lower extremity and was being followed by a vascular surgeon for lymphedema for the last 10 years. She could not tolerate paclitaxel or doxorubicin due to acute hypersensitivity reaction. She was initiated on monotherapy treatment with gemcitabine approximately 4 months before the presentation and completed six rounds of treatment. The patient remained on monthly denosumab, a RANK ligand inhibitor, for osseous metastasis. The histomorphologic features and immunohistochemistry profile of the right anterior leg soft tissue were con- 


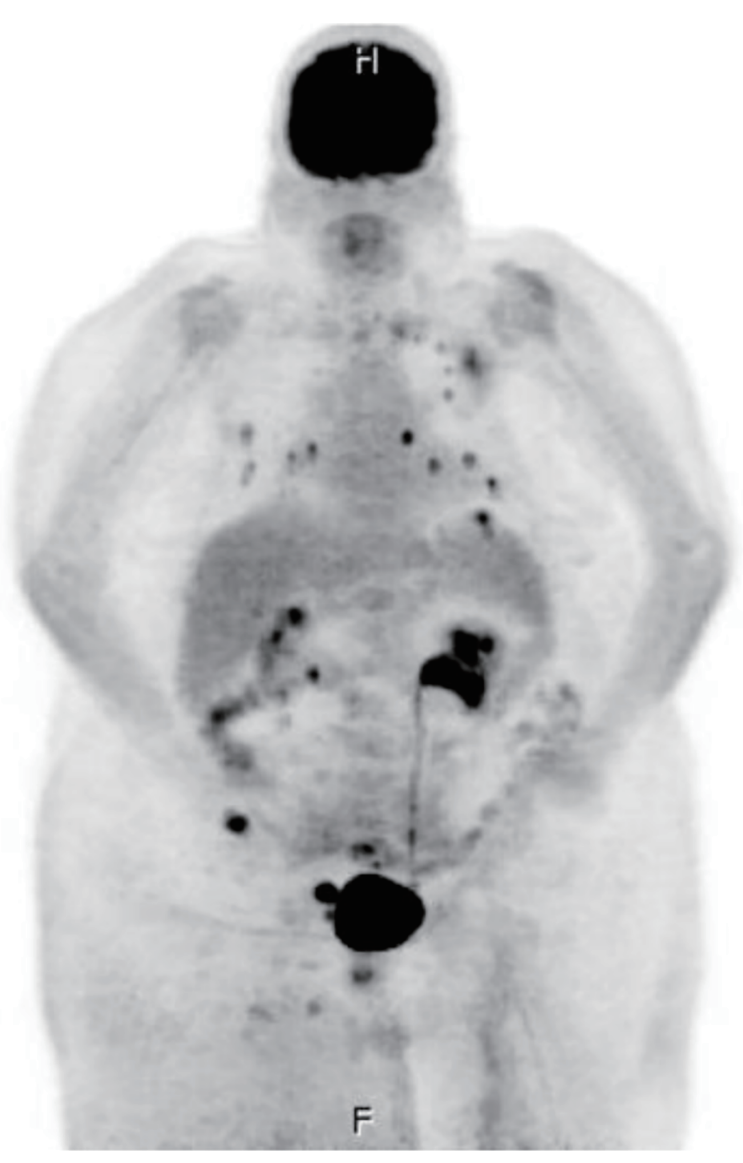

Figure 1. Whole-body positron emission tomography and computer tomography imaging were performed with multi-planar imaging without oral or intravenous contrast material, revealing metastatic disease to lymph node (right external iliac lymph node measuring $2.5 \times 4.2 \mathrm{~cm}$ ), bone (medial aspect of the left clavicle, the posterior lateral aspect of the head of the left humerus, the posterior aspect of the T2 vertebral body, and the right iliac wing), lung (there are approximately 15 solid noncalcified pulmonary nodules in each lung, ranging between 2 and $10 \mathrm{~mm}$ ), and bilateral adrenal glands.

sistent with the diagnosis of angiosarcoma with a high Ki-67 mitotic index (45\%) and with cells positive for CD34, CD31, and factor VIII (pictures not shown). The positron emission tomography (PET) scan revealed metastatic disease to lymph

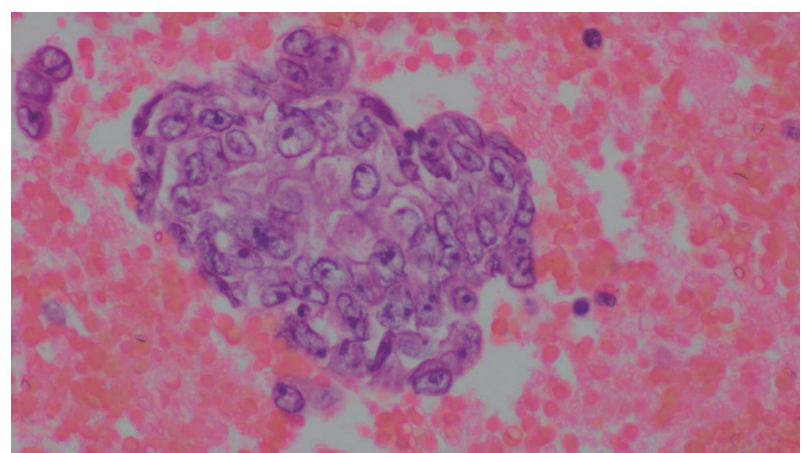

Figure 2. Pleural fluid, cell block, H\&E stain $(\times 600)$.

nodes, bone, lung, and adrenal glands (Fig. 1).

On presentation, the patient had a blood pressure of $86 / 53$ $\mathrm{mm} \mathrm{Hg}$, a pulse of 132 beats $/ \mathrm{min}$, temperature of $36.6{ }^{\circ} \mathrm{C}$, respiratory rate of 15 breaths/min, oxygen saturation $98 \%$ on bilevel positive airway pressure (Bi-PAP) with $\mathrm{FiO}_{2}$ of $60 \%$, and BMI of 41.6. On the physical exam, she was non-alert and lethargic. Rhonchi were auscultated over the lung fields bilaterally. There was minimal +1 left lower limb edema in contrast to the worse $3+$ edema of the right lower stump with serous discharge from the surgical incision consistent with wound dehiscence. There were no signs of purulence, erythema, or cellulitis associated with the wound. The rest of the physical examination was unremarkable.

\section{Diagnosis}

The complete blood count showed an elevated white blood cell count of $16.5 \times 10^{3} / \mu \mathrm{L}$ and low hemoglobin of $9.4 \mathrm{~g} / \mathrm{dL}$ (Table 1). Chest X-ray showed bilateral effusions, for which she underwent thoracentesis with pleural fluid analysis showing groups of malignant cells with positive CD34 and partial CD31 and epithelial membrane antigen positivity supporting the diagnosis of metastatic angiosarcoma (Figs. 2-5).

\section{Treatment}

The patient presented with worsening shortness of breath, primarily consistent with acute hypoxemic respiratory failure in

Table 1. Laboratory Values During Admission

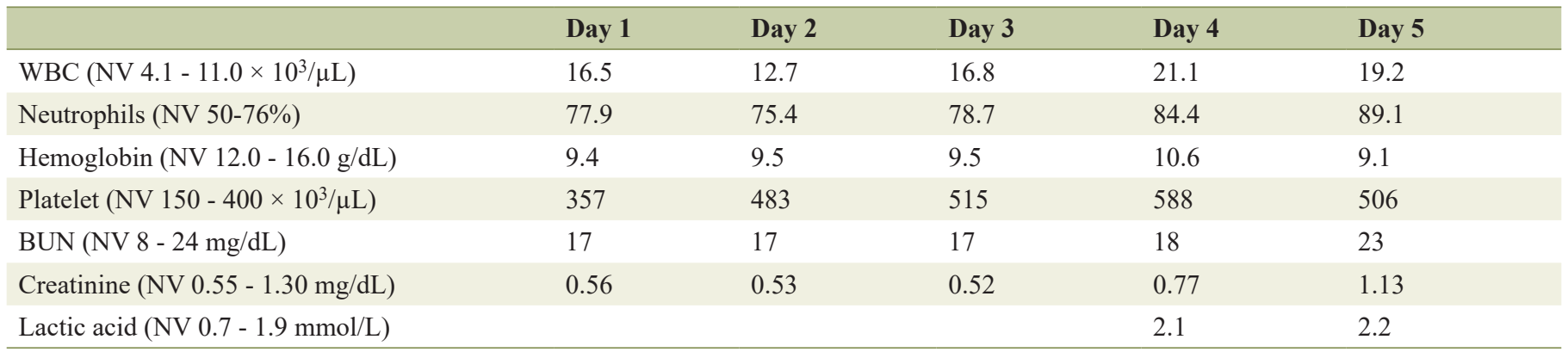

WBC: white blood cell; NV: normal value; BUN: blood urea nitrogen. 


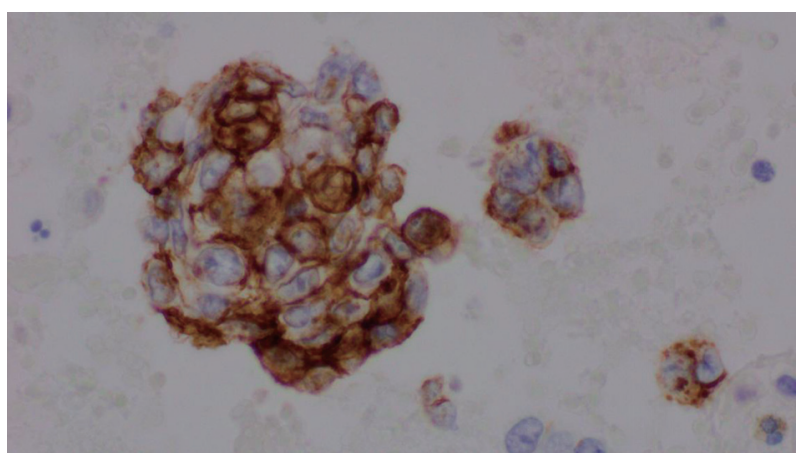

Figure 3. Pleural fluid, cell block, immunohistochemical stain for CD34 $(\times 600)$.

the setting of underlying metastatic pulmonary disease and malignant pleural effusion. She was also found to be in atrial fibrillation with a rapid ventricular response. She was subsequently given cardizem bolus and infusion and was intubated due to persistent respiratory distress. She was started on empiric antibiotic therapy with vancomycin and a combination of piperacillin and tazobactam. She continued to require ventilation support for oxygenation.

\section{Follow-up and outcomes}

The hematology-oncology service was consulted, and the patient was found to have significant progressive decompensation of underlying metastatic pulmonary disease from angiosarcoma of the right lower extremity, likely causing respiratory failure and cardiac instability. She was not a candidate for any additional therapy options for the underlying metastatic disease. The prognosis and goals of care were discussed with the patient's family and the decision was made to transition to hospice care. The patient was made comfortable and ultimately passed away in the ICU.

\section{Discussion}

Classically, STS presents after radical mastectomy in the upper extremities but can present in other areas like the lower

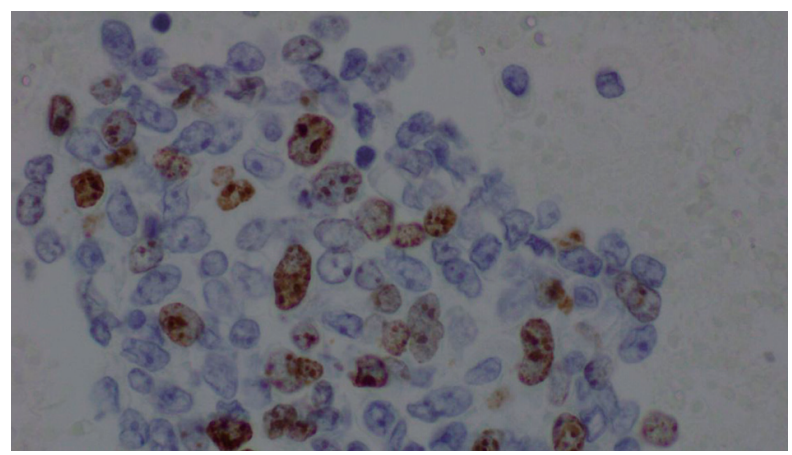

Figure 4. Pleural fluid, cell block, immunohistochemical stain for Ki-67 $(\times 600)$.

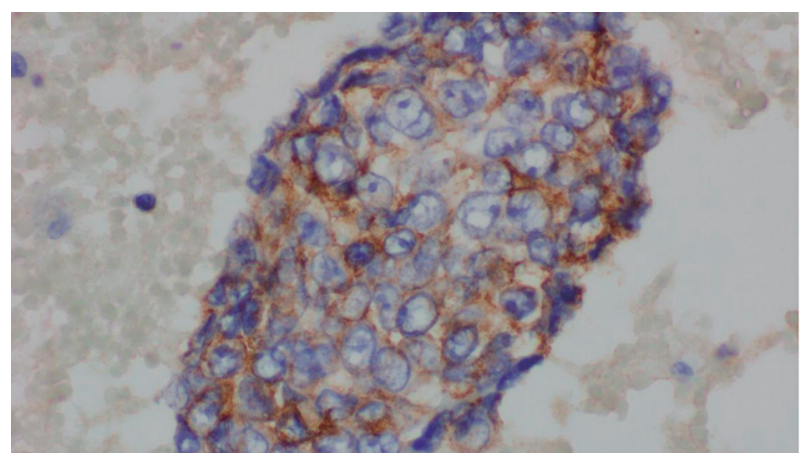

Figure 5. Pleural fluid, immunohistochemical for epithelial membrane antigen $(\times 600)$.

extremities about $10 \%$ of the time [4]. The lesions are small and typically purple at onset and then develop into a nodule. Due to these lesions' innocuous appearance, the diagnosis and treatment can be delayed [5]. Approximately 90\% of angiosarcomas associated with lymphedema occur following mastectomy. Lymphedema is a chronic, incurable condition. It is classified as primary lymphedema resulting from anomalous development of the lymphatic system or secondary lymphedema resulting from injury to the lymphatic vasculature [6]. Distant metastasis is seen in $27-42 \%$ of patients with angiosarcoma. Metastasis occurs hematogenously, most commonly to the liver, bones, soft tissues, and lymph nodes [7]. STS development in patients following radical mastectomy that survived at least 5 years is $0.07-0.45 \%$. The mechanism to which STS occurs is unknown, but it is theorized that angiogenesis in edematous regions could lead to neoplasm as collateral circulation becomes established [8]. Another theory is that chronic edema leads to localized immunodeficiency, permitting oncogenesis [2].

The incidence of chronic edema associated with obesity, called massive localized lymphedema, as seen in this patient, is trending upward with the increase in obesity in westernized societies [9]. This being said, there is a logical concern for a rise in STS incidence in these populations. Obesity-associated lymphedema in the lower extremities typically occurs once a patient's BMI reaches 50 [10]. Obesity-associated lymphedema results from the proliferation and hypertrophy of adipocytes. This hypertrophy causes lymphatic fibrosis and stasis, allowing for the accumulation of inflammatory macrophages and lymphocytes [11]. Macrophages, in particular, are known to be involved in lymphangiogenesis, producing lymphangiogenic cytokines and differentiating into lymphatic endothelial cells [12]. These macrophages encircle dead adipocytes in crown-like inflammatory foci, which release inflammatory cytokines. These cytokines are associated with increased risk for, and more aggressive behavior of, various malignancies in obese patients [13].

The overall incidence of STS has decreased with the introduction of breast-conserving therapy that removes less of the lymphatic drainage structure than radical mastectomy. Due to its rarity, the exact decrease in incidence is difficult to approximate, but since the introduction of breast-conserving therapy, the incidence of chronic lymphedema after breast cancer treat- 
ment has decreased from $40 \%$ to $4 \%$ [3].

Before starting treatment, a biopsy is required for the diagnosis of STS. On histology, angiosarcoma of STS shows a proliferation of anastomotic vascular channels that dissect collagen within the dermis. The malignant endothelial cells are commonly hyperchromatic, pleomorphic with macronuclei, and show signs of frequent mitosis. Another common feature is the presence of "hobnailing", where the endothelial cells project into the vessel lumen [14]. Staining for common endothelial cell markers such as factor VIII, anti-CD31, and antiCD34 antibodies can be useful in identifying angiosarcoma as well, with anti-CD31 being the most sensitive and specific of the three [15].

After biopsy and identification, the first-line treatment is wide tissue excision and removal of the mass. Standard adjuvant therapy is local radiation of the excision site or medical management with chemotherapy. The chemotherapeutic drugs used as the standard of care are methotrexate, 5-fluorouracil, bleomycin, or a combination of vincristine, doxorubicin, dacarbazine, cyclophosphamide, and actinomycin D [2]. There is no significant difference in survival between chemotherapy and radiation [16]. Following treatment, recurrence and metastasis are common. On initial presentation, it is thought that metastatic disease is present $16-44 \%$ of the time, and the 5 -year average survival is $40 \%$ [17]. Therefore, it is crucial to have a low biopsy threshold in patients with a newly developed skin lesion in a limb with chronic lymphedema due to the poor prognosis of STS and the benefits of early detection and treatment.

\section{Conclusion}

STS is a rare syndrome in which angiosarcoma develops on the skin in the presence of long-standing lymphedema. Although uncommon, the danger angiosarcoma poses is significant and warrants knowledge of the disease and screening in patients with chronic edema. In addition, early detection should be seen as the best way to optimize outcomes for these patients as the prognosis for metastatic angiosarcoma is poor.

\section{Acknowledgments}

We acknowledge Dr. Ward Wendy MD, Pathologist at Northwestern Hospital McHenry for the help with providing histopathology slides for the case and associated detailed description.

\section{Financial Disclosure}

None to declare.

\section{Conflict of Interest}

None to declare.

\section{Informed Consent}

Informed consent has been obtained from the patient's family.

\section{Author Contributions}

MC wrote the majority of the manuscript. XP, NT, GY, YO, and $\mathrm{AF}$ all contributed to writing and revising. $\mathrm{FZ}$ assisted in revisions of final drafts.

\section{Data Availability}

The authors declare that data supporting the findings of this study are available within the article.

\section{References}

1. Stewart FW, Treves N. Lymphangiosarcoma in postmastectomy lymphedema; a report of six cases in elephantiasis chirurgica. Cancer. 1948;1(1):64-81.

2. Young RJ, Brown NJ, Reed MW, Hughes D, Woll PJ. Angiosarcoma. Lancet Oncol. 2010;11(10):983-991.

3. Sharma A, Schwartz RA. Stewart-Treves syndrome: pathogenesis and management. J Am Acad Dermatol. 2012;67(6):1342-1348.

4. Veiga RR, Nascimento BA, Carvalho AH, Brito AC, Bittencourt Mde J. Stewart-Treves syndrome of the lower extremity. An Bras Dermatol. 2015;90(3 Suppl 1):232-234.

5. Chopra S, Ors F, Bergin D. MRI of angiosarcoma associated with chronic lymphoedema: Stewart Treves syndrome. Br J Radiol. 2007;80(960):e310-313.

6. Mehrara BJ, Greene AK. Lymphedema and obesity: is there a link? Plast Reconstr Surg. 2014;134(1):154e-160e.

7. Gaballah AH, Jensen CT, Palmquist S, Pickhardt PJ, Du$\operatorname{ran}$ A, Broering G, Elsayes KM. Angiosarcoma: clinical and imaging features from head to toe. Br J Radiol. 2017;90(1075):20170039.

8. Murgia RD, Gross GP. Stewart-Treves Syndrome. In: StatPearls. Treasure Island (FL), 2021.

9. Berebichez-Fridman R, Deutsch YE, Joyal TM, Olvera PM, Benedetto PW, Rosenberg AE, Kett DH. StewartTreves syndrome: a case report and review of the literature. Case Rep Oncol. 2016;9(1):205-211.

10. Greene AK, Grant FD, Maclellan RA. Obesity-induced Lymphedema Nonreversible following Massive Weight Loss. Plast Reconstr Surg Glob Open. 2015;3(6):e426.

11. Zampell JC, Aschen S, Weitman ES, Yan A, Elhadad S, De Brot Andrade M, Mehrara BJ. Regulation of adipogenesis by lymphatic fluid stasis: part I. Adipogenesis, fibrosis, and inflammation. Plast Reconstr Surg. 2012;129(4):825834.

12. Maruyama K, Ii M, Cursiefen C, Jackson DG, Keino H, Tomita M, Van Rooijen N, et al. Inflammation-induced lymphangiogenesis in the cornea arises from CD11b-positive macrophages. J Clin Invest. 2005;115(9):2363-2372. 
13. Howe LR, Subbaramaiah K, Hudis CA, Dannenberg AJ. Molecular pathways: adipose inflammation as a mediator of obesity-associated cancer. Clin Cancer Res. 2013;19(22):6074-6083.

14. Lee R, Saardi KM, Schwartz RA. Lymphedema-related angiogenic tumors and other malignancies. Clin Dermatol. 2014;32(5):616-620.

15. McHaffie DR, Kozak KR, Warner TF, Cho CS, Heiner JP, Attia S. Stewart-Treves syndrome of the lower extremity.
J Clin Oncol. 2010;28(21):e351-352.

16. Grobmyer SR, Daly JM, Glotzbach RE, Grobmyer AJ, 3rd. Role of surgery in the management of postmastectomy extremity angiosarcoma (Stewart-Treves syndrome). J Surg Oncol. 2000;73(3):182-188.

17. Buehler D, Rice SR, Moody JS, Rush P, Hafez GR, Attia $\mathrm{S}$, Longley BJ, et al. Angiosarcoma outcomes and prognostic factors: a 25-year single institution experience. Am J Clin Oncol. 2014;37(5):473-479. 